\title{
coulabilité des matériaux agro-alimentaires
}

\author{
flowability of food products
}

\author{
M.-P. LUONG \\ CNRS-LMS Ecole Polytechnique*
}

Rev. Franç. Géotech. n 52, pp. 37-49 (juillet 1990)

\section{Résumé}

Le stockage en silo des produits granulaires en vrac génère toute une classe inhabituelle de problèmes de rupture à la fois structurale et fonctionnelle principalement dus à la méconnaissance de la rhéologie de la très grande variété des matériaux ensilés. La connaissance de leur comportement rhéologique autorise une meilleure estimation des efforts et des pressions sur les parois. Les méthodes actuelles de dimensionnement sont souvent basées sur les hypothèses des grains constitutifs rigides et d'un comportement macroscopique parfaitement plastique, seulement valables pour une catégorie particulière de matériaux. Cette communication propose une caractérisation de quelques produits agro-alimentaires en paramètres rhéologiques obtenus sur trois types de trajet de chargement. La notion de seuil caractéristique ou seuil de désenchevêtrement de la structure granulaire s'est révélée tout à fait adaptée, si ce n'est indispensable pour analyser le degré de coulabilité des matériaux ensilés.

\footnotetext{
Abstract

Silo storage of bulk materials generates an unusual class of both structural and functional failure problems, mainly caused by a misunderstanding of the rheology of the great variety of stored materials. The knowledge of their rheological behaviour permits a better evaluation of loads and stresses on the silo walls. The design methods in use are often based on simplified assumptions such as rigid grains and perfect plasticity for the macroscopic behaviour, only valid for a particular type of bulk materials. This paper proposes a rheological characterization of some granular food products on three types of loading path using the conventional triaxial cell. The characteristic state concept or disaggregation threshold of the granular structure reveals to be quite suitable and indispensable for examining the flowability of the stored bulk materials.
} 


\section{INTRODUCTION}

Le stockage en silo des produits granulaires en vrac génère toute une classe inhabituelle de problèmes de rupture à la fois structurale (pliage, flambage, fissuration, voire fendage et effondrement des parois) et fonctionnelle (formation des voûtes, des cavités, de cheminée ou de noyau au sein du matériau ensilé et surtout difficulté, voire impossibilité d'extraire le produit stocké)

Les estimations des efforts et des pressions sur les parois du silo sont souvent incertaines, hasardeuses et généralement basées sur des notions empiriques simplificatrices parfois peu évidentes, vu la très grande diversité des matériaux en vrac à ensiler.

L'objet de cette étude est tout d'abord une détermination expérimentale du comportement rhéologique des matériaux ensilés sur différents trajets de chargement simulant les conditions de remplissage et de vidange du silo, en vue d'obtenir des paramètres utilisables dans une modélisation numérique.

En même temps, lanalyse des mécanismes de déformation de ces matériaux tout au long du chargement permet de préciser le concept d'un état caractéristique d'enchevêtrement ou dé désenchevêtrement de la structure granulaire par rapport au seuil d'écoulement maximal. Cette approche rhéologique, tant au niveau granulaire qu'au niveau macroscopique, présente l'avantage d'interpréter à la fois le mécanisme de mobilisation des efforts dans les parois du silo et les phénomènes de coulabilité extrêmement importants pour le choix d'un organe d'extraction « ad hoc ».

Nous nous proposons d'examiner le comportement rhéologique de quelques matériaux agro-alimentaires comme du blé, du riz en paddy, des haricots ou du colza à l'appareil triaxial cylindrique sous différents trajets de chargement, à confinement radial constant et à contrainte moyenne constante.

\section{CALCULS DES PRESSIONS DANS LES SILOS}

Les charges qu'exercent la matière stockée dans les silos ont fait l'objet d'études, de recherches et d'essais $[3,4,11,12,22]$ dans le monde entier depuis que l'homme a cherché à emmagasiner des produits en vrac en vue des périodes moins propices à la production.

Les méthodes conventionnelles de détermination du champ de contraintes dans les silos sont généralement basées sur l'équilibre limite d'un milieu bidimensionnel rigide plastique avec un critère de rupture sans tenir compte des caractéristiques rhéologiques des matières ensilées. Utilisant des notions d'états actif ou de poussée et passif ou de butée de Rankine, et négligeant les conditions aux limites, de nombreux auteurs ont essayé de façon classique de déterminer, sans convaincre, l'état de contrainte sur la paroi. La méthode des lignes caractéristiques déduites des équations d'équilibre et du critère de rupture n'est pas plus convaincante dans l'hypothèse de déformation plane, car les trois équations du problème mathématique suffisent à déterminer les trois inconnues qui décrivent l'état de contrainte à l'équilibre limite au sein des matériaux ensilés, quelle que soit leur déformabilité pourvu qu'ils aient le même critère de rupture. Ces diverses méthodes donnent des résultats numériques ne dépendant que du poids volumique, du frottement interne des matériaux ensilés et de la rugosité relative de la paroi du silo. Elles sont donc tout à fait utiles dans les cas où ces différents paramètres suffisent à caractériser l'état rhéologique du matériau ensilé sans trop s'écarter de la réalité.

L'existence des forces de frottement de la matière ensilée sur les parois s'opposant aux forces de gravité du produit a amené JANSSEN dès 1895 à formuler la théorie de voûte [11] applicable au silo vertical de section constante et de longueur indéfinie, rempli de matière pulvérulente incompressible. Cette méthode simplificatrice considère l'équilibre statique d'une tranche horizontale de matériau pesant et frottant sur les parois du silo. Elle constitue la base des techniques conventionnelles de dimensionnement des silos. Par la suite, CAQUOT et KÉRISEL [4] ont donné une solution mécaniquement plus cohérente. Ces diverses méthodes utilisent la notion d'un rayon moyen au sens hydraulique égal au rapport de l'aire transversale au périmètre, c'est-à-dire admettant implicitement un écoulement homogène en masse du matériau ensilé.

Expérimentalement de nombreuses études tant à l'étranger qu'en France ont montré que les pressions sur les parois varient avec le temps et que les opérations de vidange de quantité infime de produit changent les valeurs des efforts dans des proportions très importantes. Il en est de même au cours des processus de fonctionnement du silo en remplissage ou en vidange. Les sollicitations exercées sur les parois dépendent de la forme, du fond et du mode d'écoulement à la vidange et d'après la position des orifices. En outre, il a été très facile de constater que la distribution des efforts varie d'une façon extrême s'il s'agit de matériaux compressibles comme les matériaux agro-alimentaires, ou de matériaux dilatants comme le charbon pulvérisé, le ciment, la chaux ou le gypse, ou les minerais durs à gros grains anguleux. Ces constatations expérimentales mettent en évidence linfluence déterminante du comportement rhéologique des matériaux en vrac à ensiler dans la détermination des efforts à considérer pour le dimensionnement d'un silo.

\section{3. ÉTAT CARACTÉRISTIQUE D'UN MATÉRIAU GRANULAIRE}

L'analogie du comportement rhéologique des métaux et des sols, caractérisée par le palier de charge sur la courbe contrainte-déformation (fig. 1), a suggéré le 


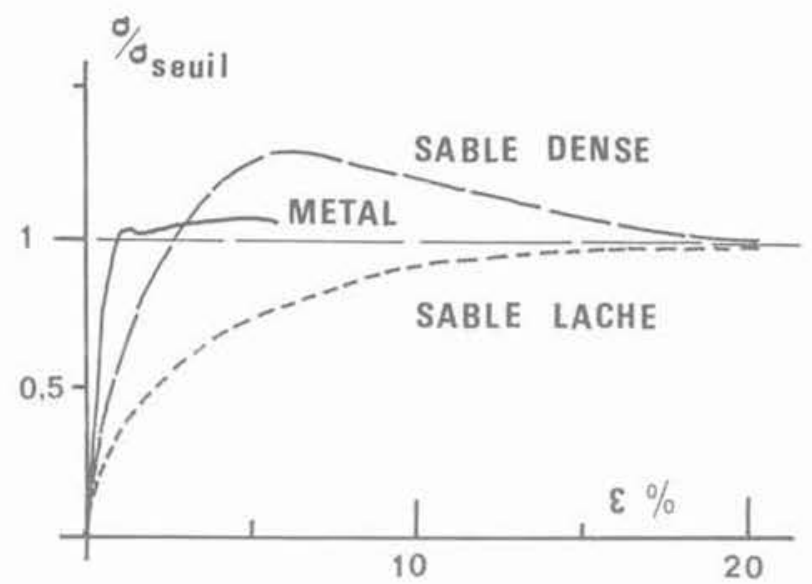

Fig. 1. - Analogie de comportement métal-sol. Fig. 1. - Similarity of metal-soil behaviour.

concept d'un état idéalisé du sol appelé « Etat Critique s [21] pour modéliser le comportement des sols sableux ou argileux à l'état ultime qu'ils peuvent atteindre en grandes distorsions lors de la rupture à volume constant.

La théorie de la dilatance [20] pour des matériaux frottants est fondée sur une approche différente à partir d'une modélisation du glissement relatif des grains formant la structure du matériau, dans le domaine des premières déformations et jusqu'à la rupture. Cette approche du comportement des sables se base en particulier sur une écriture du bilan d'énergie qui se réalise lors d'un chargement monotone dans les conditions de symétrie de lappareil triaxial classique de révolution. Elle associe, directement à une inclinaison des contraintes définie par l'angle de frottement mobilisé, une vitesse de déformation volumique nulle sous cisaillement. Elle généralise ainsi le résultat utilisé dans la théorie de l'état critique pour décrire l'état ultime permanent du matériau frottant.

Ces deux approches du comportement des sols sableux sous chargement monotone, directement reliées à la déformation plastique des matériaux, prennent naissance à partir d'une modélisation du comportement de ces matériaux dans le domaine des grandes déformations, soit à l'état ultime, soit principalement dans la phase de dilatance, au voisinage d'un maximum éventuel de chargement.

Pour rendre compte du comportement des matériaux au cours des processus de stockage en silo au remplissage ou à la vidange, il est nécessaire d'approfondir ce premier stade d'étude en s'intéressant aux mécanismes de déformation couplés à la fois en volume et en distorsion du matériau tout au long de l'histoire du chargement, et non seulement à la rupture en grandes déformations. En effet, au cours des opérations de remplissage et de vidange, les déformations dépendent de l'état de contrainte, de l'anisotropie induite lors des chargements antérieurs et, surtout des mécanismes d'enchevêtrement et de désenchevêtrement à rigidité volumique imposée par les dimensions du silo, qui autorisent ou non la coulabilité des matériaux ensilés, nécessaire pour une extraction aisée.

\subsection{Mécanismes de déformation}

Un matériau granulaire en vrac comme le blé, le riz en paddy, le haricot ou le colza peut être considéré comme un assemblage de grains en contact neutre les uns avec les autres: le tout forme une structure globale créée à partir de la superposition des éléments de base présentant des propriétés physiques particulières: texture relative au degré de finesse et d'uniformité du matériau, sphéricité ou formes allongées, rugosité, adhérence, accrochage par des liaisons en nombre variable avec la contrainte moyenne, com. pressibilité, etc.

Le comportement mécanique de ces matériaux ne peut s'expliquer que par cette propriété constitutive et de ce fait à deux niveaux différents :

- d'une part, au niveau du grain élémentaire où la liaison grain-à-grain dépend essentiellement de la déformabilité du matériau, de sa texture superficielle, de sa susceptibilité au taux d'humidité et de sa forme;

- d'autre part, au niveau de la structure formée par l'ensemble des grains pouvant se grouper en agrégats ou parcelles plus ou moins orientés.

Quand les particules d'un agrégat stable n'adhèrent pas les unes aux autres, elles sont disposées suivant une structure élémentaire dans laquelle chaque grain est en contact avec plusieurs de ses voisins. Suivant l'arrangement, une structure élémentaire peut être plus ou moins compacte. Les matériaux à éléments fins peuvent être stables même lorsque les grains sont en contact en très peu de points, pourvu que l'adhérence entre les grains soit du même ordre de grandeur que le poids des grains: c'est le cas de la structure en nid d'abeilles. Les grains les plus gros sont parfois capables de former un squelette dont les interstices sont remplis par un agglomérat relativement meuble des constituants plus fins du matériau : cet arrangement donne une structure en squelette. Il existe des structures mottées formées de mottes élémentaires plus compactes. Les grains plats ou allongés peuvent former des structures fortement anisotropes. Les particules filiformes présentent une structure souvent lâche, mais à enchevêtrement maximal. Enfin, certains matériaux constitués de grains identiques polygonaux sont caractérisés par un arrangement susceptible aux blocages géométriques.

Ces deux niveaux, microscopique et macroscopique, ne sont pas totalement indépendants: leurs propriétés fondamentales devraient être exprimées au niveau le plus restreint, c'est-à-dire en fonction des propriétés d'un grain élémentaire en contact avec ses voisins. Une telle approche repose sur la modélisation des contacts intergranulaires. La forme allongée des particules favorise des mécanismes de flexion des grains qui se traduisent au niveau global par une très forte compressibilité quasi réversible (fig. 2).

Les déformations irréversibles globales proviennent essentiellement des modifications de la structure du matériau étudié. Pour schématiser ces principaux mécanismes de déformation, il est possible d'envisager principalement : 


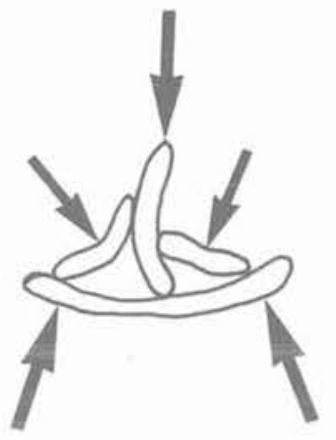

TRÈS CHARGÉ

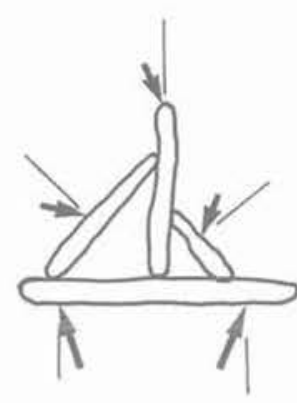

\section{PEU CHARGÉ}

Fig. 2, - Mécanismes de déformation réversible des particules oblongues entraînant une diminution de l'angle d'incidence des forces de contact.

Fig. 2. - Mechanisms of reversible deformation of oblong particules inducing a decrease of incidence angle of contact forces.

- un serrage des grains les uns sur les autres;

- un déplacement relatif de ces grains les uns par rapport aux autres, associé le plus souvent à une rotation de ceux-ci :

- une déformation des particules;

- un écrasement des grains ;

- une modification physico-chimique du produit agro-alimentaire par variation d'ambiance hygrométrique ou thermique.

Dans un domaine de contrainte limité, la rupture ou l'écrasement des grains d'un matériau siliceux en compression est peu probable ou non significative ; celle des grains agro-alimentaires, par contre, est probable, due à une localisation des efforts. Les mécanismes prépondérants de déformation irréversible seront donc constitués par le serrage des grains, en particuler lors de la phase initiale de chargement du matériau, et essentiellement par le réarrangement des grains lors des déplacements relatifs dans le cas d'un changement de trajet de sollicitation. La rotation des grains susceptible d'accompagner le réarrangement de la structure peut être associée à une réorientation de celleci ; elle peut créer dans un échantillon une anisotropie de structure induite par la déformation.

Les mécanismes de réarrangement des grains, de caractère purement géométrique, peuvent induire dans le matériau des déformations volumiques irréversibles importantes, en particulier lors d'une phase de dilatance lorsque les forces de glissement qui agissent sur les grains deviennent suffisantes pour provoquer un désenchevêtrement de la structure du matériau.

\subsection{Procédure expérimentale}

Ces différents mécanismes de comportement rhéologique peuvent être examinés à l'aide des essais triaxiaux à symétrie de révolution avec les notations suivantes (fig. 3) :

$$
\begin{array}{ll}
\text { Contrainte moyenne } & \mathrm{p}=\left(\sigma_{1}+2 \sigma_{3}\right) / 3 \\
\text { Déviateur } & \mathrm{q}=\sigma_{1}-\sigma_{3}
\end{array}
$$

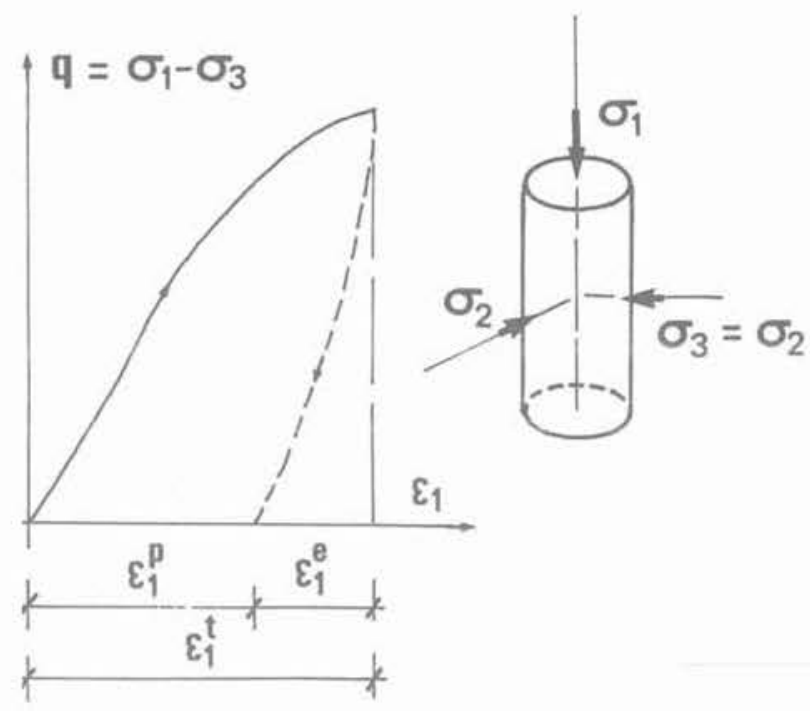

Fig. 3. - Paramètres de l'essai triaxial cylindrique

Fig. 3. - Parameters of the cylindrical triaxial test.

Niveau déviatorique $\eta=\mathrm{q} / \mathrm{p}$

auxquelles on associe les paramètres de déformation :

volumique

distorsionnelle

$\epsilon_{\mathrm{v}}=\epsilon_{1}+2 \epsilon_{3}$

$\epsilon_{\mathrm{q}}=2\left(\epsilon_{1}-\epsilon_{3}\right) / 3$

taux de dilatance

$\delta=\Delta \epsilon_{\mathrm{v}} / \Delta \epsilon_{\mathrm{q}}$

L'incrément de travail dû au tenseur contrainte $\sigma$ et au tenseur incrément de déformation $\dot{\epsilon}$ peut alors s'écrire :

$$
\dot{W}=\sigma: \dot{\epsilon}=(\mathrm{p} I+\mathrm{s}):\left(\dot{\epsilon}_{\mathrm{v}} \mathrm{I}+\dot{e}\right)
$$

L'incrément de travail total $\dot{W}$ se sépare donc en partie volumétrique $W_{v}$ et une partie déviatorique $W_{s}$, $s$ et e étant respectivement les tenseurs déviateurs de contrainte et de déformation. L'incrément de travail volumétrique s'écrit de façon unique comme le produit des invariants du tenseur de contrainte et du tenseur incrément de déformation. Moyennant certaines hypothèses simplificatrices sur l'incrément de déformation sur un trajet triaxial cylindrique où la déformation élastique est relativement faible, l'incrément de travail irréversible dissipé peut s'écrire :

$$
\dot{W}^{1}=p \dot{\epsilon}_{v}{ }^{1}+q \dot{\epsilon}_{q}{ }^{1}
$$

Les essais ont été réalisés en compression triaxiale avec accourcissement axial et/ou en extension triaxiale avec allongement axial à vitesse de charge $\dot{\sigma}_{1}$ constante ou à vitesse de déformation $\dot{\epsilon}_{1}$ constante.

Avec la notation $\sigma_{1} \geq \sigma_{2} \geq \sigma_{3}$, nous remarquons un saut du trièdre des contraintes principales entre les états de compression et d'extension. Les chemins de contrainte explorés sont donc localisés dans des plans méridiens à symétrie ternaire du trièdre des contraintes. Pratiquement ces chemins de contrainte correspondent, au voisinage de l'axe du silo, aux trajets de chargement au sein du matériau ensilé lors des pro- 
cessus de remplissage (une contrainte principale majeure et deux contraintes principales mineures égales) et de vidange (deux contraintes principales majeures égales et une contrainte principale mineure). Le critère de Mohr-Coulomb s'écrit pour un sol pulvérulent sans cohésion:

$$
\mathrm{f}(\sigma)=\operatorname{Max}\left[\sigma_{1}(1-\sin \phi)-\sigma_{1}(1+\sin \phi)\right]
$$

où $\phi$ est l'angle de frottement interne et $\mathrm{i}, \mathrm{j}=1$ à 3. A la rupture de l'échantillon, les niveaux déviatoriques en compression $\eta_{f}^{+}$et extension $\eta_{f}$ triaxiales s'écrivent respectivement :

$$
\begin{aligned}
\eta_{t}^{+} & =6 \sin \phi_{f}^{+} /\left(3-\sin \phi_{f}^{+}\right) \\
\text {et } \eta_{f} & =6 \sin \phi_{\hat{f}} /\left(3+\sin \phi_{f}\right)
\end{aligned}
$$

Les valeurs correspondant à l'état caractéristique en compression ou en extension triaxiale s'obtiennent en remplaçant l'indice $f$ par l'indice $c$.

Les conditions opératoires imposées à l'échantillon d'élancement (hauteur sur diamètre) $H / D \geq 2$ à 2,5 sont :

(a) $\sigma_{2}=\sigma_{3}=$ cte, c'est le cas de l'essai triaxial conventionnel à pression de confinement constante,

(b) $\eta=$ cte sur ce chemin radial dans l'espace des contraintes, l'obliquité moyenne des contraintes reste constante, (c) $\mathrm{p}=$ cte, la contrainte moyenne est maintenue constante en faisant varier la pression de confinement avec la charge axiale.

Le chargement monotone à vitesse de charge constante jusqu'à l'écoulement plastique permet de suivre l'évolution des mécanismes de déformation du matériau avec le niveau de contrainte ainsi que l'influence du trajet de charge sur ces mécanismes.

Le chargement cyclique à un ou plusieurs niveaux de contrainte moyenne et de contrainte déviatorique avec un faible nombre de cycles quasi statiques (par exemple vingt cycles au maximum pour chaque niveau) nous renseigne sur le caractère pseudo-réversible du matériau étudié et permet la détermination des paramètres physiques capables de décrire son comportement dans une modélisation numérique.

\subsection{Essais triaxiaux conventionnels}

Lors d'un essai triaxial cylindrique en compression $\sigma_{1}>\sigma_{2}=\sigma_{3}=$ cte. le matériau subit initialement une diminution de volume : avec les notations des mécaniciens des sols, la déformation volumique est donc d'abord positive. Lorsque la contrainte déviatorique $\mathrm{q}$ appliquée à l'échantillon croît, la vitesse de déformation volumique tend à s'annuler, dans le cas d'un matériau lâche, pour des déformations axiales très importantes. Pour un matériau plus dense initialement, la vitesse de déformation volumique s'inverse et le matériau connaît alors une phase de dilatance avant d'atteindre le domaine des grandes déformations (fig. 4).

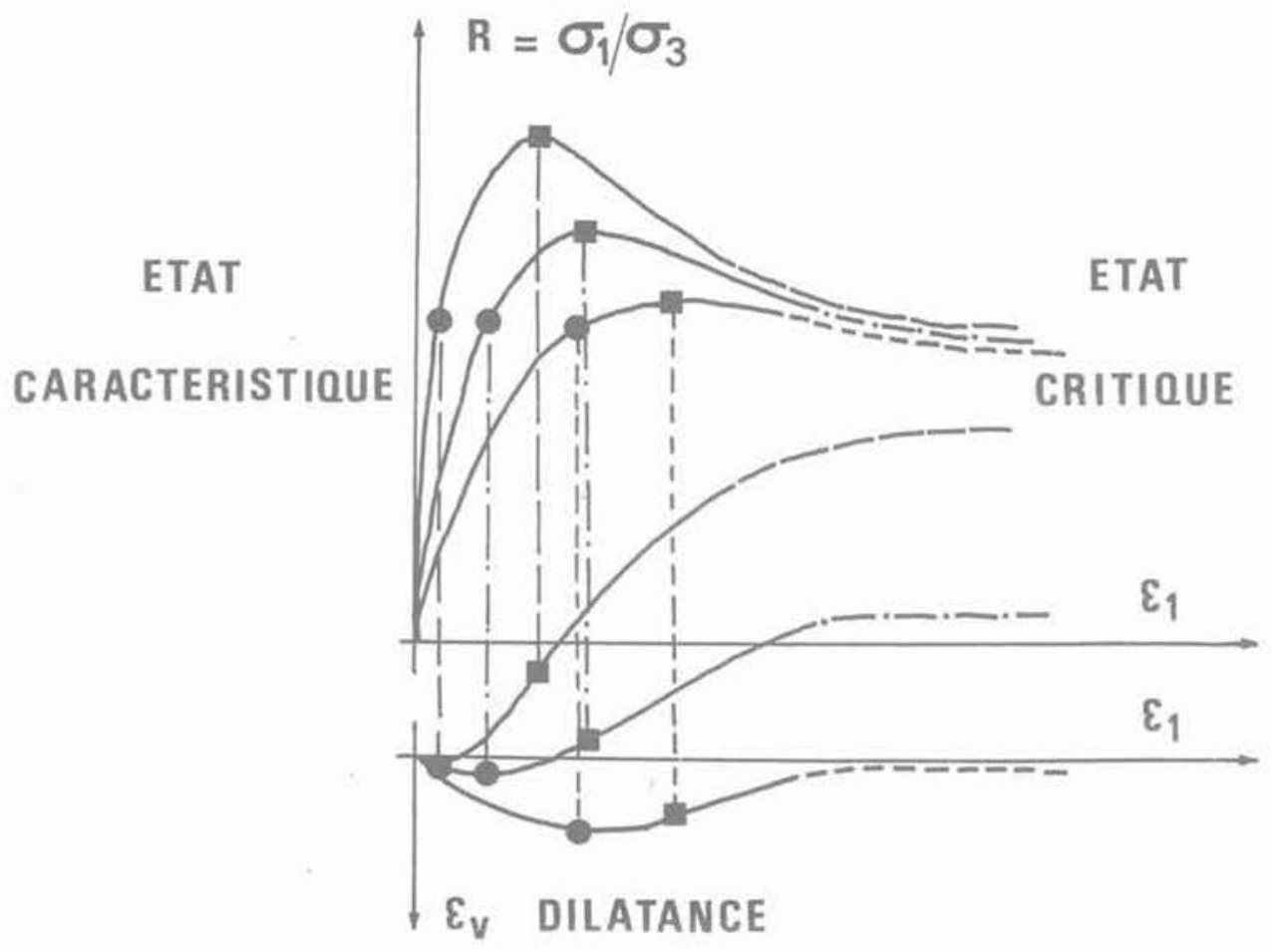

Fig. 4. - Etats critique et caractéristique par rapport

à la théorie de la dilatance des sols.

Fig. 4. - Comparison of critical, characteristic states and dilatancy theory of soils. 
Cette propriété a suggéré la définition d'un angle analogue à un angle de frottement caractérisant le comportement des agrégats [13] à ce niveau suivant linterprétation classique de Coulomb. Cet angle particulier vérifie la propriété commune mise en évidence à l'état critique et dans le cadre de la dilatance des matériaux pulvérulents : pour une inclinaison des contraintes équivalente à cet angle de frottement, la vitesse de déformation volumique du matériau est nulle. Pour des valeurs courantes de lordre de $30^{\circ}$, l'angle de frottement critique mobilisé après le pic dé chargement sur du sable est voisin de cet angle de frottement mis en évidence avant la rupture. De nombreux auteurs ont admis la coïncidence de ces angles dans leur modélisation du comportement du sol [23] bien qu'il n'en soit rien pour des matériaux à fort angle de frottement comme le feldspath [15].

Les essais en extension $\sigma_{1}=\sigma_{2}>\sigma_{3}$ correspondant au trajet de charge en phase de vidange des silos conduisent à des résultats semblables. Le matériau est initialement contractant, puis dilatant. Ces essais permettent de la même façon d'évaluer un angle de frottement interne associé au changement de sens de la déformation volumique que le matériau subit sous chargement monotone. La valeur de cet angle de frottement en extension peut être différente de celle obtenue dans le cas d'un chargement en compression pour certains matériaux. Elle les caractérise.

\subsection{Critère caractéristique}

En utilisant simplement le niveau déviatorique de contrainte atteint par le matériau lors de l'inversion de sens des déformations volumiques entre les phases de contractance et de dilatance, il est donc facile de définir un « état caractéristique des matériaux pulvérulents \& où les propriétés suivantes sont vérifiées :

- la vitesse de déformation volumique est nulle $\left(\dot{\epsilon}_{\mathrm{v}}=0\right)$;

- le niveau de contrainte atteint par le matériau définit un angle de frottement caractéristique $\phi_{c}$ qui détermine le seuil de désenchevêtrement du matériau [16] permettant au matériau pulvérulent de s'écouler.

Ces deux propriétés de contractance et de dilatance sont classiquement associées lors d'un chargement analogue à volume contrôlé : le seuil caractéristique doit correspondre dans ces conditions au seuil de contrainte où se produisent les phénomènes de désenchevêtrement permettant l'écoulement des matériaux ensilés lors de la vidange.

Expérimentalement, quelles que soient les conditions de chargement, on peut observer une boucle de dilatance dessinée par le paramètre $\epsilon_{\mathrm{v}}$ variation de volume suivie d'une contractance rapide du matériau à la décharge lorsque le seuil caractéristique est franchi : ce dernier est ainsi clairement indiqué par l'apparition de cette boucle de dilatance (fig. 5). Cette boucle de dilatance $B D$, qui se forme dans un diagramme $\left(q, \epsilon_{\mathrm{v}}\right)$ ou $\left(\eta, \epsilon_{\mathrm{v}}\right)$ après franchissement du seuil caractéristique lors de la décharge, est un indicateur fidèle de la présence du seuil caractéristique autorisant le désenchevêtrement de la matière ensilée.
Les essais réalisés sur d'autres trajets de chargement, en compression comme en extension, confirment que cette propriété de formation d'une boucle de dilatance détermine de façon précise la position du seuil caractéristique dans l'espace de contrainte.

Une procédure de détermination de la valeur du paramètre $\eta_{c}$ ou seuil caractéristique de désenchevêtrement pour un matériau donné, peut donc être définie à partir de cette propriété indépendamment du trajet de chargement suivi, en effectuant des cycles successifs de charge jusqu'à des valeurs maximales croissantes de l'inclinaison des contraintes, pour mettre en évidence la boucle de dilatance qui finit par se former au franchissement du seuil.

Cette approche phénoménologique, basée sur le concept de l'état caractéristique, met en évidence :

- limportance du trajet de contrainte qui favorise, selon les cas, l'un des mécanismes de déformation associé à des déformations volumiques importantes, soit à des déformations de distorsions prépondérantes :

- P'écrouissage qui traduit la différence de comportement entre le chargement initial sur un matériau vierge et les cycles suivants de décharge-recharge; - la présence d'hystérésis plus ou moins accentuée traduisant l'existence ou non d'une modification du réseau de contacts accompagnée ou non d'une compressibilité significative des grains constitutifs ;

- l'anisotropie induite par le chargement, dépendant de la nature morphologique des grains.

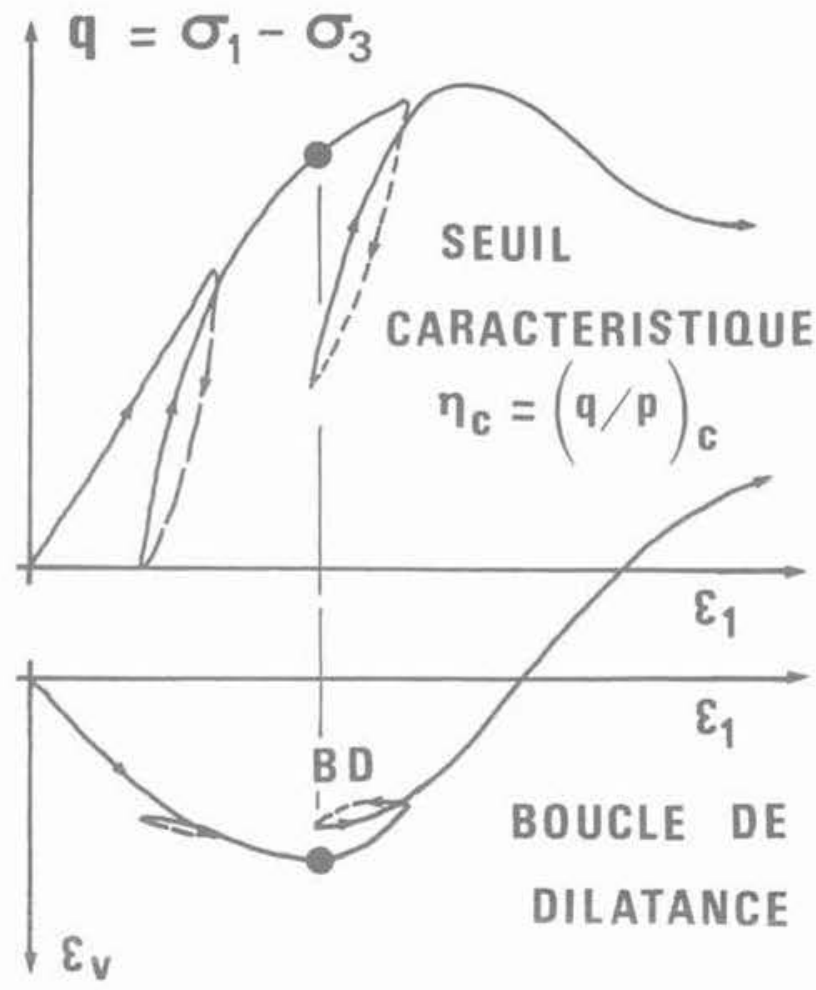

Fig. 5. - Définition đu seuil caractéristique. Fig. 5. - Definition of the characteristic threshold. 


\section{CARACTÉRISTIQUES RHÉOLOGIQUES DES MATÉRIAUX TESTÉS}

Les essais ont été effectués sur des éprouvettes de $70 \mathrm{~mm}$ de diamètre et d'élancement supérieur à 2 . Les courbes effort-déformation enregistrées en continu pendant le chargement donnent pour chaque essai la variation de volume $\Delta \mathrm{V} / \mathrm{V}_{0}$ par rapport au volume initial et le déplacement axial $\Delta \mathrm{h} / \mathrm{h}_{0}$ par rapport à la hauteur initiale, en fonction de la charge axiale $\Delta \mathrm{F} / \mathrm{S}_{0}$ où $\mathrm{S}_{0}$ est la section initiale de l'échantillon.
Suivant le matériau et la pression de confinement, on peut distinguer deux types de comportement au cours du chargement :

- une contraction volumique suivie d'une dilatation continue jusqu'à l'écoulement du matériau ;

- une contraction continue jusqu'à l'écoulement.

L'analyse des courbes expérimentales (fig. 6) montre que pour un matériau donné, les deux types de comportement ne se manifestent pas par hasard, mais présentent un passage continu suivant la pression de confinement de l'essai : le phénomène de dilatance dis-

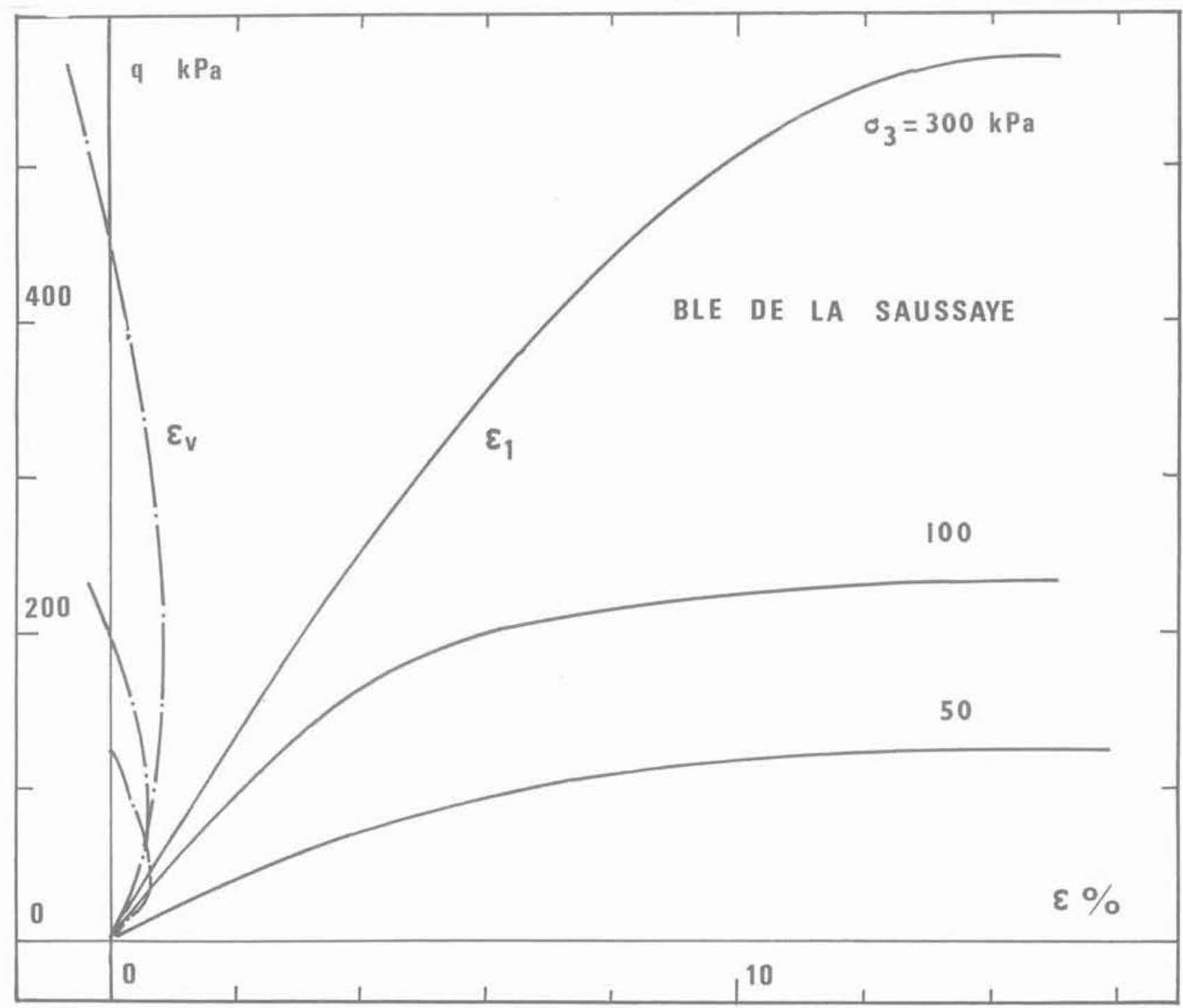

Fig. 6a. - Comportement rhéologique du blé de Saussaye sous confinement constant.

Fig. 6a. - Rheological behaviour of Saussaye wheat under constant confining pressure. 


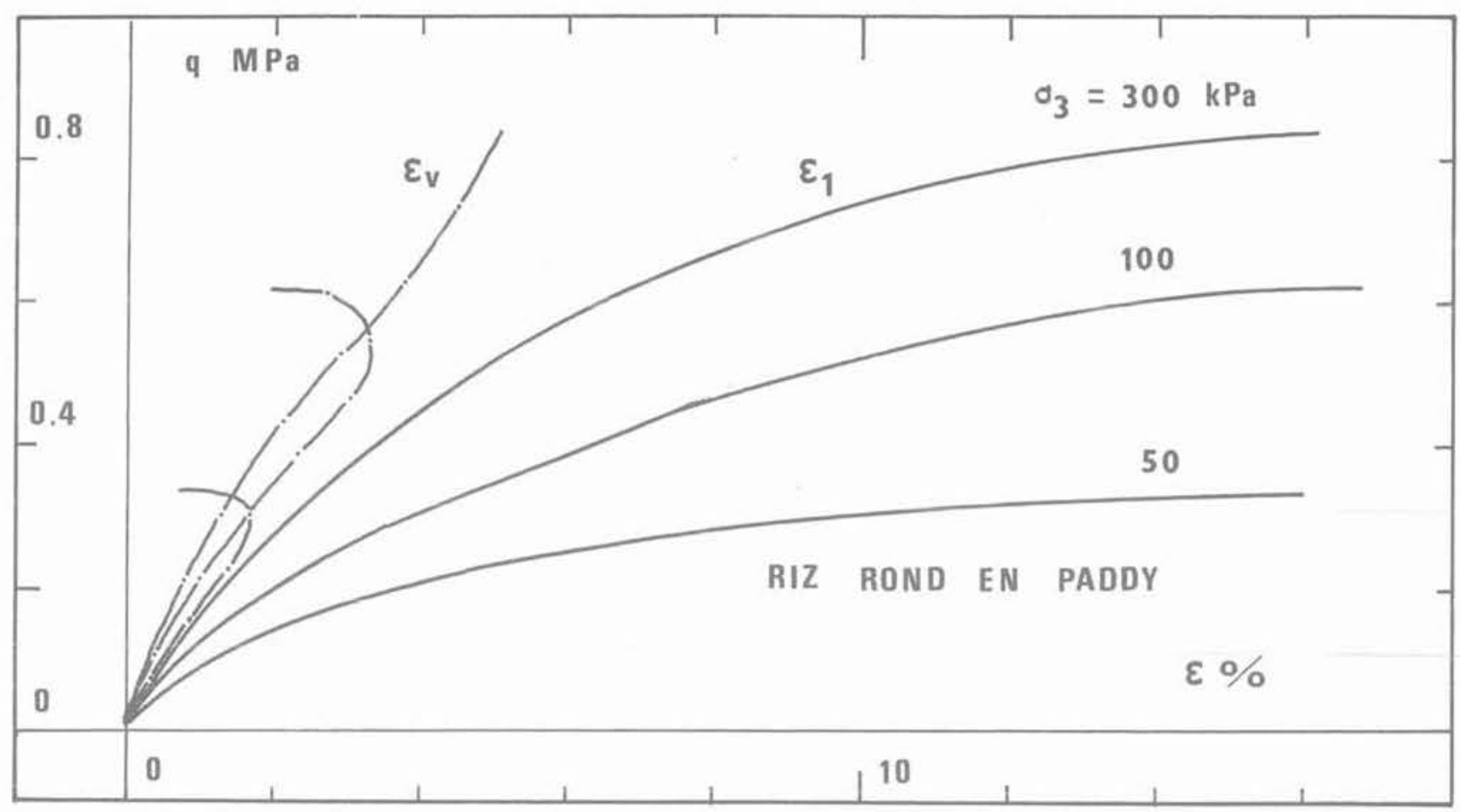

Fig. 6b. - Riz rond en paddy sous confinement constant.

Fig. 6b. - Paddy round rice under constant confining pressure.

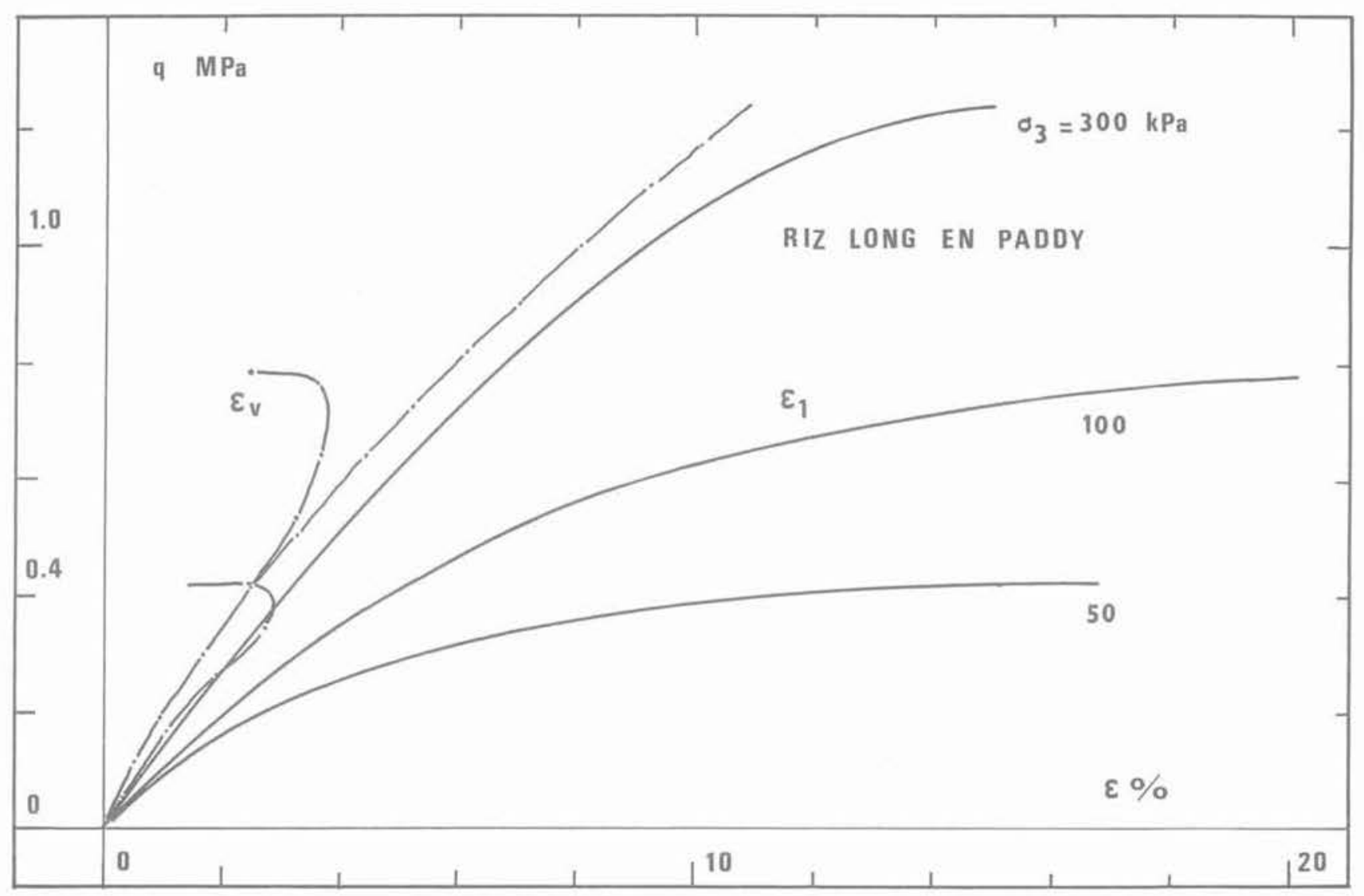

Fig. 6c. - Riz long en paddy sous confinement constant.

Fig. 6c. - Paddy long rice under constant confining pressure. 


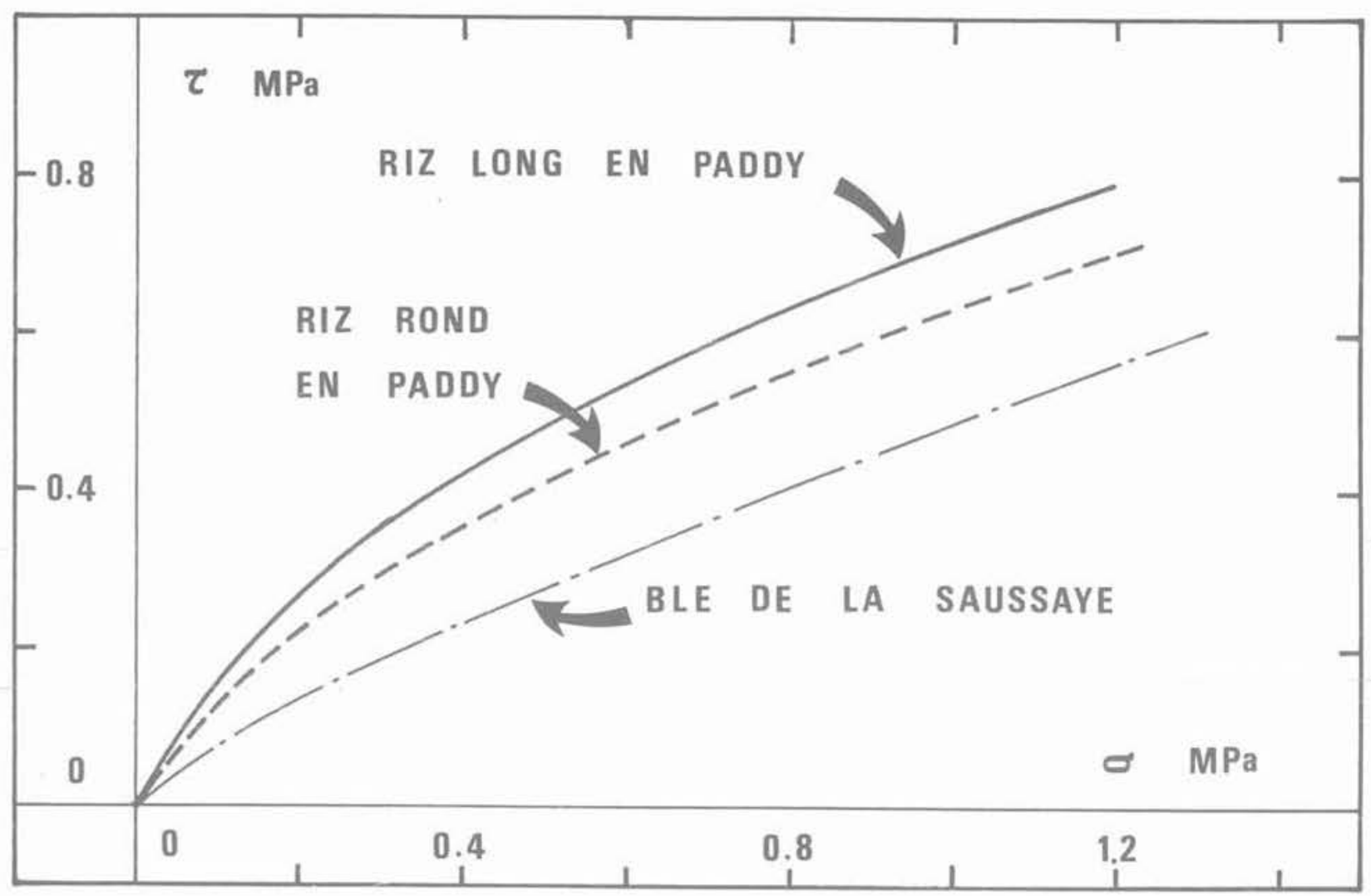

Fig. 7. - Courbes intrinsèques des matériaux étudiés.

Fig. 7. - Intrinsic curves of materials in study.

paraît lorsque la pression de confinement augmente et empêche le désenchevêtrement des grains.

Les principaux résultats obtenus sur les matériaux testés : blé, riz en paddy, haricot ou colza peuvent être traduits en valeurs d'angles de frottement $\phi_{f}^{+}, \phi \frac{f}{\text {, }}$ $\phi_{c}^{+}$et $\phi_{c}^{-}$qui sont calculées avec l'interprétation de Coulomb pour un matériau pulvérulent au pic de contrainte, au seuil caractéristique, en compression et en extension.

Les courbes intrinsèques de certains matériaux testés sont non linéaires et plutôt de type parabolique (fig. 7). Les valeurs manquantes pour le seuil caractéristique signifient que le matériau ne présente pas de seuil caractéristique jusqu'au niveau de contrainte imposé. Autrement dit, le désenchevêtrement granulaire ne peut pas s'opérer pour ces cas de chargement avec comme conséquence pratique : la coulabilité est mauvaise, voire impossible, pour le matériau soumis à ce niveau de contrainte.

\section{COMPORTEMENT SUR TRAJET RADIAL}

Sur le trajet de chargement radial, le rapport $\eta=q / p$ ou niveau déviatorique est maintenu constant. On peut constater qu'à partir d'un état initial, toute sollicitation isotrope et/ou déviatorique provoque aussi- tôt des déformations irréversibles. Bien que l'état de contrainte " macroscopique " du chargement soit isotrope, on peut imaginer que de nombreux contacts entre grains soient voisins de l'équilibre limite. Une modification de l'état de contrainte initial, aussi petite soit-elle, entraîne le glissement de ces grains. Au cours d'un premier chargement, les déformations irréversibles entraînent une modification de la structure de l'échantillon par réarrangement des grains. Le matériau s'adapte à la sollicitation appliquée : c'est l'écrouissage. Cet écrouissage crée autour du chemin suivi, lors du premier chargement, un domaine où le matériau présente un comportement presque réversible.

Expérimentalement, (fig. 8) on constate que les essais radiaux cycliques permettent de mettre en évidence plus facilement la réversibilité du matériau testé :

- au cours de ces essais, les déformations plastiques irréversibles diminuent rapidement avec le nombre de cycles ;

- la courbe de charge-décharge devient une courbe fermée si le rapport $\eta$ est inférieur au seuil caractéristique ;

- la relation contrainte-déformation à la décharge est pratiquement identique à elle-même, tout au long des cycles de chargement.

Cette caractéristique de la décharge radiale vis-à-vis du comportement à la recharge a été reconnue par de nombreux chercheurs $[2,7,9,17]$. 


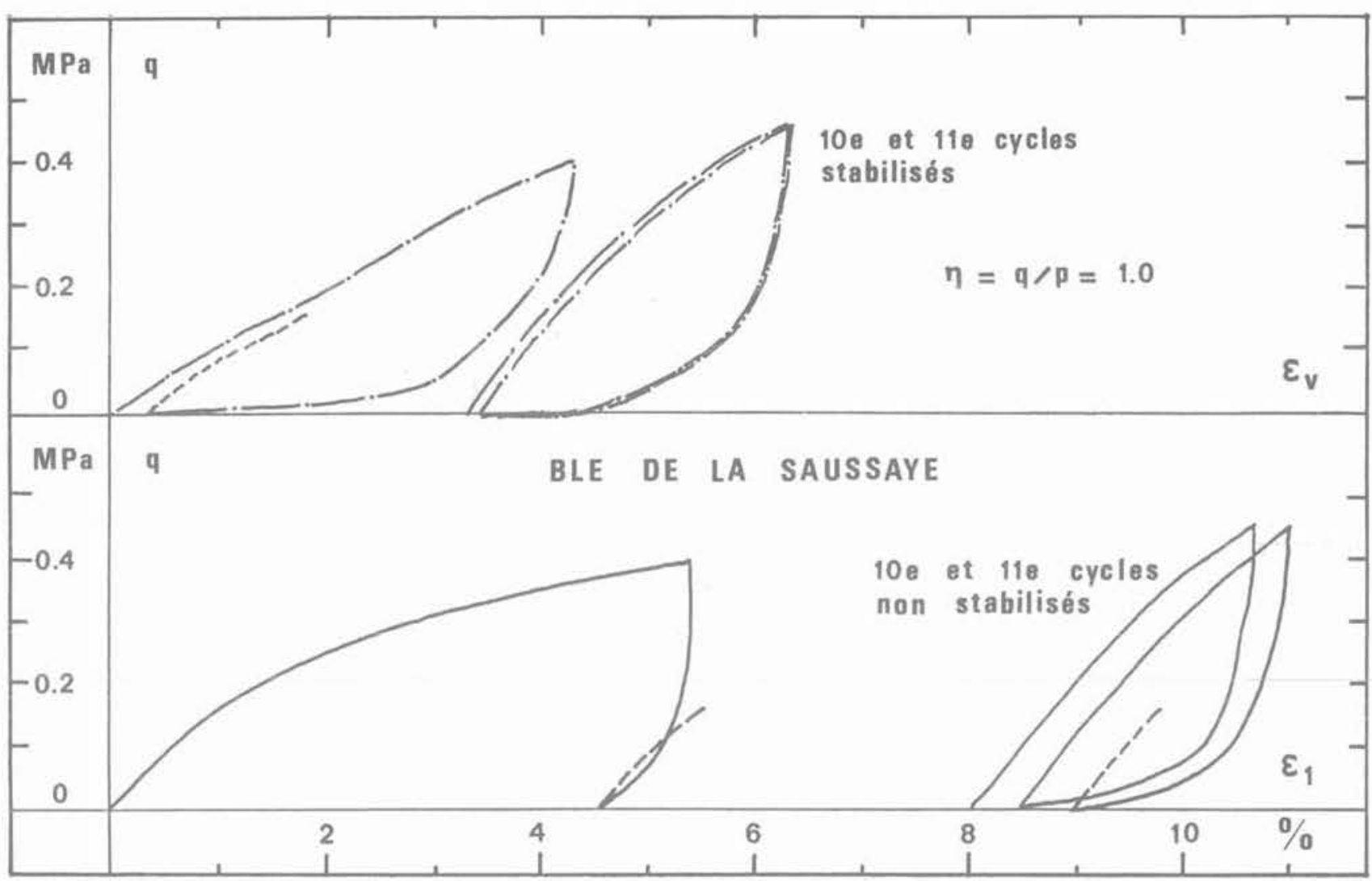

Fig. 8. - Blé de Saussaye sous chargement radial.

Fig. 8. - Saussaye wheat under radial loading.

\section{COMPORTEMENT A CONTRAINTE MOYENNE CONSTANTE}

L'ensemble des résultats présentés a illustré linfluence très significative de la contrainte moyenne sur le comportement rhéologique du matériau, aussi bien au niveau granulaire relatif au frottement, à la compressibilité, à la cinématique du grain qu'au niveau global concernant le seuil de désenchevêtrement ou de la résistance maximale. Il est donc nécessaire d'examiner également la réponse rhéologique du matériau sur le trajet de charge à contrainte moyenne constante qui privilégie la mise en évidence des phénomènes de contractance et de dilatance du squelette granulaire.

Le trajet de chargement à contrainte moyenne $\mathrm{p}$ constante est obtenu à lappareil triaxial cylindrique en faisant varier simultanément et linéairement la pression de confinement et le déviateur de contrainte à partir d'un état de contrainte isotrope.

Les résultats expérimentaux obtenus sur le trajet à p constante (fig. 9) confirment les conclusions des essais sur le trajet conventionnel à confinement constant.

\section{INTÉRÊT DE LA CARACTÉRISATION PAR L'ESSAI TRIAXIAL}

Pour le stockage en silo de produits en vrac, le problème industriel se pose en termes d'exigences à imposer aux produits, aux silos et aux éléments d'extraction pour garantir un déroulement non perturbé des opérations.

L'essai triaxial conventionnel est apte à reproduire les états de contrainte et les trajets de charge subis par les matériaux au cours des processus d'ensilage. Il permet de déterminer d'une façon relativement simple la réponse rhéologique qui conditionne l'écoulement des matériaux lors de la vidange du silo. En outre l'expérience constatée sur des silos en service montre qu'il existe divers modes d'écoulement du produit ensilé [14].

\section{Ecoulement en cheminée}

* Le produit n'est extrait qu'en forme d'un trou de rat dans une zone très limitée au-dessus de la sortie. Le produit se trouvant contre les bords reste dans le silo ». 


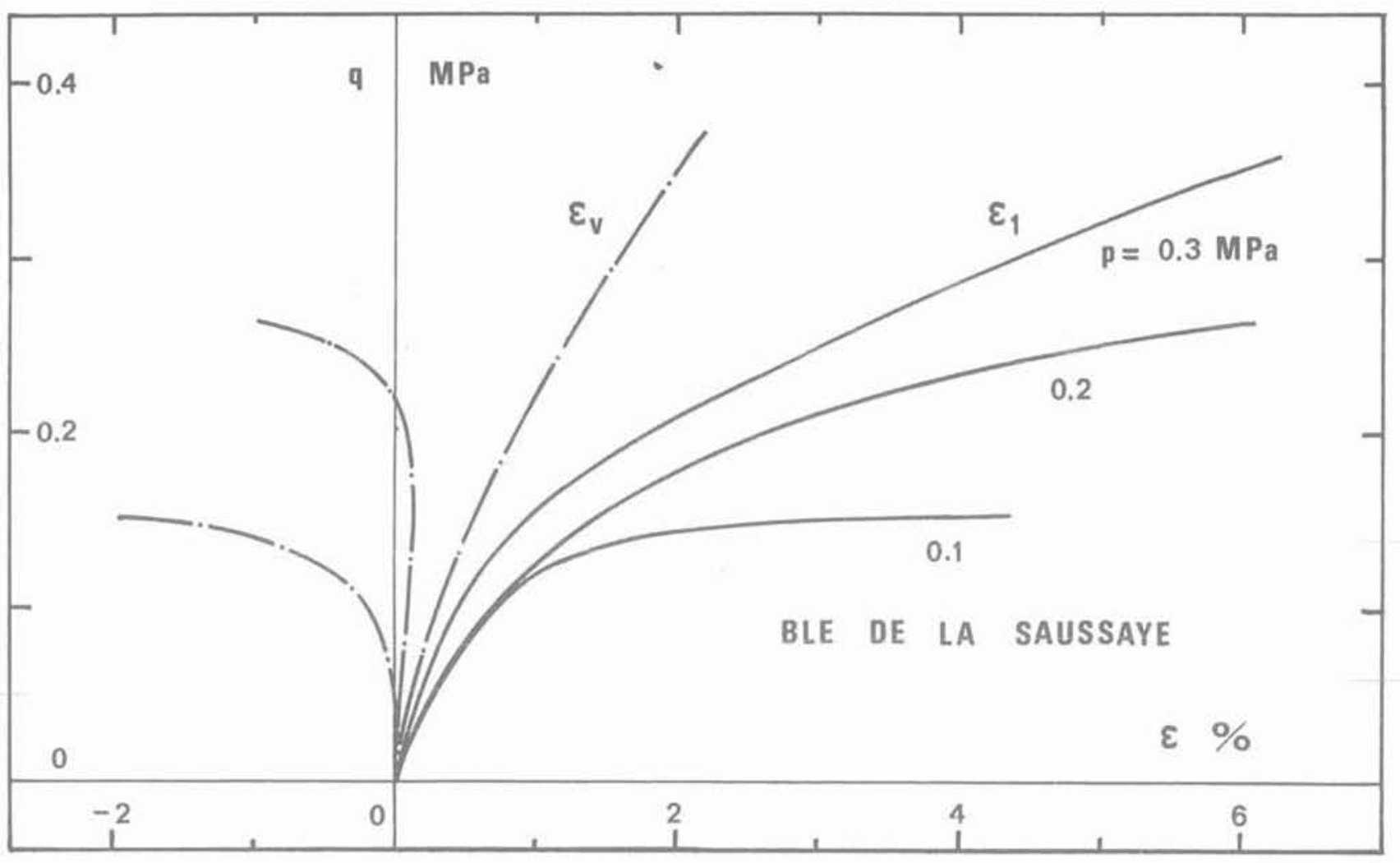

Fig. 9. - Blé de Saussaye sous chargement à contrainte moyenne constante.

Fig. 9. - Saussaye wheat under constant mean stress loading.

Ce type d'écoulement pourrait se manifester pour un matériau peu compressible présentant une résistance maximale très supérieure au seuil caractéristique de désenchevêtrement: $q_{\max } / q_{c}$ très grand et $\mid \epsilon_{\mathrm{vmax}}-$ $\epsilon_{\mathrm{vc}}$ très faible.

\section{Ecoulement en noyau}

* Seule une zone centrale est en mouvement lors de l'extraction, les zones extérieures restent d'abord immobiles et s'éboulent en partant du haut quand la vidange est plus avancée ».

$\mathrm{Ce}$ mode suggèrerait que le produit présente également une résistance maximale supérieure au seuil de désenchevêtrement, mais beaucoup plus cormpressible et dépendant d'une manière significative de la contrainte moyenne. Ce qui permet une propagation des zones en mouvement du haut (où la contrainte moyenne est initialement faible) vers le bas par suite d'une redistribution progressive des contraintes au sein de la matière ensilée.

\section{Formation de voûte}

«L'extraction est interrompue par une voûte de produit, il faut tisonner pour que l'écoulement continue .

La formation d'une voûte bloquant l'écoulement du matériau ensilé est due à une absence du mécanisme de désenchevêtrement de la structure granulaire. Le seuil caractéristique du matériau n'est pas atteint au sein du matériau dans la partie formée en voûte.

\section{Ecoulement en masse}

« Toute la masse du produit ensilé se met en mouvement lors de la vidange $»$.

C'est le cas où la résistance maximale au cisaillement et le seuil caractéristique sont relativement faibles et du même ordre de grandeur. L'incompressibilité du matériau entraîne un mouvement homogène et rapide de tout le volume, alors qu'une certaine déformabilité de la matière favorise un écoulement plus progressif de toute la masse.

Ces constatations expérimentales suggèrent de prendre en compte sur un essai triaxial cyclindrique classique :

1. un indice de désenchevêtrement $I_{d}$ défini (fig. 10) par le rapport du travail $W_{\mathrm{e}}$ d'enchevêtrement sur le travail $W_{\mathrm{d}}$ de dilatance globale à la rupture: le désenchevêtrement de la structure granulaire s'opèrera d'autant plus facilement que cet indice $I_{d}$ est faible. 2. un indice de coulabilité $I_{c}$ défini (fig. 10) par le rapport du travail $W_{\text {de }}$ de distorsion jusqu'au seuil d'enchevêtrement au travail $W_{d m}$ mobilisé jusqu'à la résistance au cisaillement maximale du matériau : la coulabilité en masse du matériau est d'autant meilleure que cet indice $I_{c}$ est faible.

Il est ainsi facile de comprendre que les modes d'écoulement des matériaux ensilés lors des opérations 
$I_{d}=\frac{W_{e}}{W_{M}}=\frac{\int^{\varepsilon_{v c}} p d \varepsilon_{v}}{\int^{\varepsilon_{v M}}\left|p d \varepsilon_{v}\right|} \quad I_{c}=\frac{W_{d e}}{W_{d M}}=\frac{\int^{\varepsilon_{q c}} q d \varepsilon_{q}}{\int_{q M}^{\varepsilon_{q} d d \varepsilon_{q}}}$

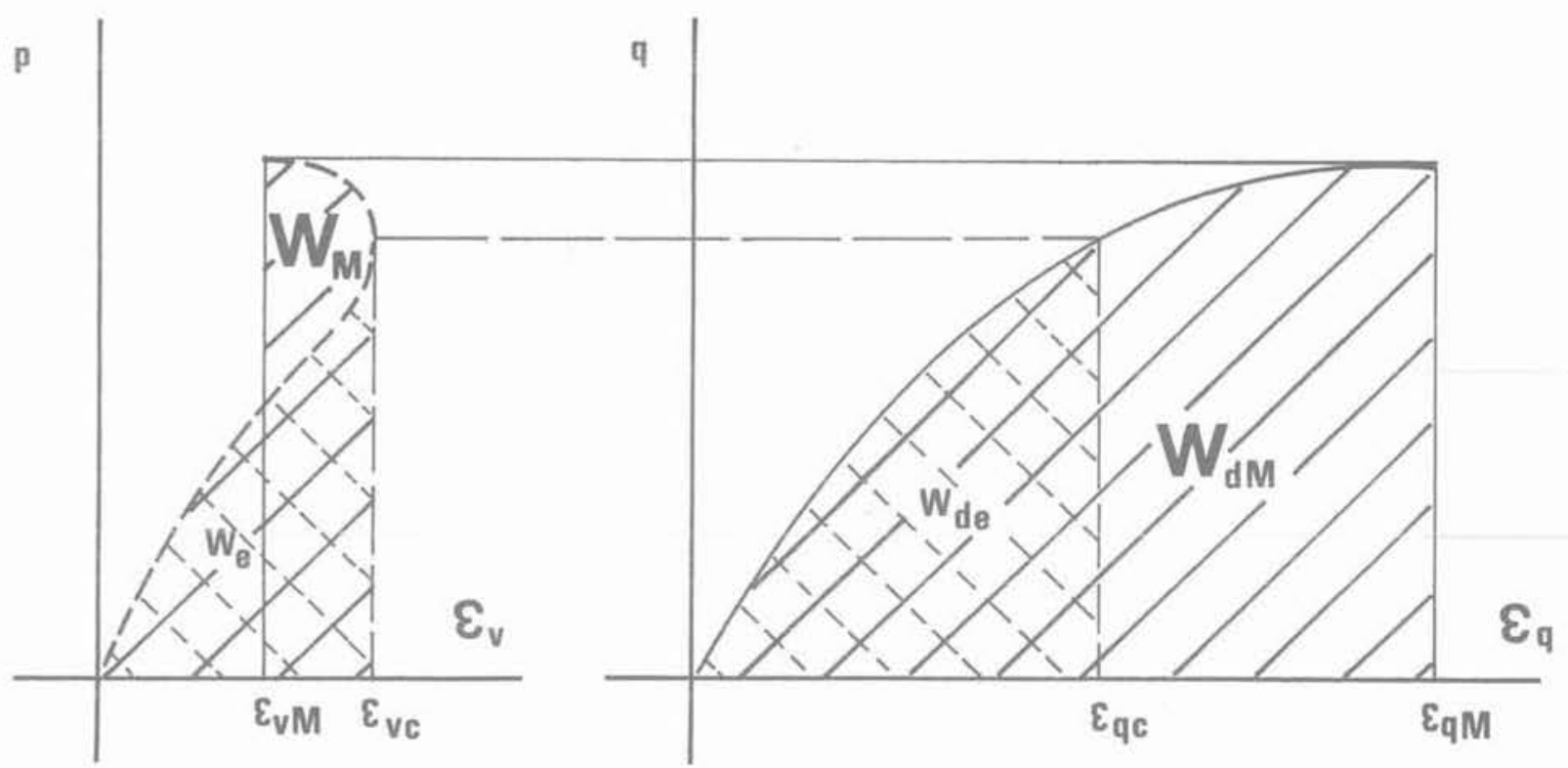

Fig. 10. - Définition des indices de désenchevêtrement $I_{d}$ et de coulabilité $I_{c}$ Fig. 10. - Definition of dissaggregation and flowability indexes.

de vidange dépendent à la fois de leurs paramètres de résistance (frottement interne maximal et frottement interne de désenchevêtrement) et de leurs paramètres de déformabilité (compressibilité à la résistance maximale et au seuil de désenchevêtrement). Les tests classiques de coulabilité $[5,6]$ en tiennent plus ou moins compte et paraissent adaptés pour des matériaux relativement légers comme les poudres fluidisables. C'est en négligeant l'effet de la contrainte moyenne sur le frottement interne et la compressibilité qu'ils deviennent inopérants dans les cas où la pesanteur joue un rôle relativement important, surtout lorsque le matériau présente une courbe intrinsèque non linéaire d'allure fortement parabolique.

\section{CONCLUSION}

Cette étude expérimentale a permis d'examiner le comportement rhéologique de quelques produits agroalimentaires (blé, riz de paddy, petit pois et colza) sur trois trajets de charge.

La notion d'état caractéristique des sols sableux s'est révélée tout à fait adaptée pour définir le seuil de désenchevêtrement de la structure granulaire et analyser les conditions de coulabilité des matériaux agroalimentaires ensilés.

Les indices de désenchevêtrement $\mathrm{I}_{d}$ et de coulabilité $I_{c}$ globalisant les états de contrainte et de défor- mation sont des indicateurs tout à fait utiles pour prévoir l'éventualité de formation d'effet de voûte ainsi que les modes d'écoulement des matériaux ensilés au cours des opérations de vidange.

Il serait extrêmement intéressant de pouvoir vérifier sur des cas réels si les assertions découlant de l'état caractéristique sont justifiées pour les modes d'écoulement dans les silos à la vidange.

\section{REMERCIEMENTS}

Cette étude a été encouragée par le Ministère de la Recherche et de la Technologie, et soutenue par la Commission des Silos Métalliques.

\section{BIBLIOGRAPHIE}

[1] BABIC M., SHEN H.H. (1989), " Simple mean free path theory for stresses in a rapid granular flow ", ASCE J. Engng Mech., 115, n 6 , $1262-1282$.

[2] BARDEN L., KHAYATT A.J. (1966), " Incremental strain and strength of sand in the triaxial test ", Géotechnique, 16, 4, 338-367.

[3] BLIGHT G.E. (1986), "Pressures exerted by materials stored in silos ", Géotechnique, 36, $n^{\circ} 1,33-56$. 
[4] CAQUOT A., KERISEL J. (1949), «Traité de Mécanique des Sols „, Gauthier-Villars, Paris.

[5] CARR R.L. (1965), "Evaluating flow properties of solids ", Chemical Engineering, 163-168, January 1965.

[6] DEGOUTTE G. (1989), "Caractérisation en laboratoire des propriétés physiques et mécaniques des matières ensilées », Construction Métallique, 2, 91-95.

[7] EL SOHBY M.A. (1989), “Deformation of sands under constant stress ratios". Proc. 7th ICSMFE, Mexico, 111-119.

[8] GOODMAN M.A., COWIN S.C. (1971), "Two problems in the gravity flow of granular materials", J. Fluid Mech., 45, 321-339.

[9] HARDIN B.O. (1978), "The nature of stress. strain behaviour of soils ", Proc. Conf. on Earthquake Engng. and Soil Dynamics, Pasadena, ASCE Pub., 1, 3-90, June, 19-21.

[10] HAUSSLER U., EIBL J. (1984), "Numerical investigations on discharging silos ”, ASCE J. Engng Mech., 110, n 6, 957-971.

[11] JANSSEN H.A. (1985), *Versuche über Gedreidedruck in Silozellen », Z. Ver. Dt. Ing., 1, 1045-1050.

[12] JENIKE A.W. (1964), "Steady gravity flow of frictional-cohesive solids in converging channels ", ASME J. Appl. Mech., 86, 5-11.

[13] KIRKPATRICK W.M. (1961), "Discussion on soil properties and their measurement". Proc. 5th ICSMFE Paris, III, 131-133, Dunod.

[14] KUHNEMUND B. (1987), «Stockage en silos de produits en urac», Extrait de Bühler BühlerMiag Nouvelles 217.

[15] LEE I.K. (1966), "Stress-dilatancy performance of feldspar ", J. Soil Mech. Found. Div., ASCE, 92, SM2, 79-103.

[16] LUONG M.P. (1978), « Etat caractéristique du sol », CR Acad. Sci., 287, B, 305-307, Paris.

[17] LUONG M.P. (1980), " Stress-strain aspects of cohesionless soils under cyclic and transient loading ", Proc. ISSCTL, 315-324, A.A. BALKEMA, Rotterdam, The Netherlands.

[18] Mc TIGUE D.F. (1982), "A non linear constitutive model for granular materials: application to gravity flow", ASME, J. Appl. Mech., 49, 291-296.

[19] PASQUARELL G.C., ACKERMAN N.L. (1989), "Boundary conditions for planar granular flows ", ASCE J. Engng Mech., 115, n 6 , 1283-1301.

[20] ROWE P.W. (1971), "Theoretical meaning and observed values of deformations for soils, stressstrain behaviour of soils », Cambridge, 143-194, ed R.G.H. Parry.

[21] SCHOFIELD A.N., WROTH C.P. (1986), "Critical state soil mechanics". Mc Graw Hill, London, UK.

[22] WALKER D.M. (1966), "An approximate theory for pressures and arching in hoppers", Chemical Engineering Science, 21, 975.

[23] WROTH P., BASSET R.H. (1965), "A stressstrain relationship for the shearing behaviour of a sand", Géotechnique, 1, 32-56, UK. 